\title{
Binding constant of cell adhesion receptors and substrate-immobilized ligands depends on the distribution of ligands
}

\author{
Long $\mathrm{Li}^{1}{ }^{1}$ Jinglei $\mathrm{Hu},{ }^{2,3,{ }^{*}}$ Guangkui $\mathrm{Xu},{ }^{4}$ and Fan Song ${ }^{1,5, \dagger}$ \\ ${ }^{1}$ State Key Laboratory of Nonlinear Mechanics (LNM) and Beijing Key Laboratory of Engineered Construction and Mechanobiology, \\ Institute of Mechanics, Chinese Academy of Sciences, Beijing 100190, China \\ ${ }^{2}$ Kuang Yaming Honors School, Nanjing University, Nanjing 210023, China \\ ${ }^{3}$ Shenzhen Institute of Research, Nanjing University, Shenzhen 518057, China \\ ${ }^{4}$ School of Aerospace, Xi'an Jiaotong University, Xi'an 710049, China \\ ${ }^{5}$ School of Engineering Science, University of Chinese Academy of Sciences, Beijing 100049, China
}

(Received 2 November 2017; published 16 January 2018)

\begin{abstract}
Cell-cell adhesion and the adhesion of cells to tissues and extracellular matrix, which are pivotal for immune response, tissue development, and cell locomotion, depend sensitively on the binding constant of receptor and ligand molecules anchored on the apposing surfaces. An important question remains of whether the immobilization of ligands affects the affinity of binding with cell adhesion receptors. We have investigated the adhesion of multicomponent membranes to a flat substrate coated with immobile ligands using Monte Carlo simulations of a statistical mesoscopic model with biologically relevant parameters. We find that the binding of the adhesion receptors to ligands immobilized on the substrate is strongly affected by the ligand distribution. In the case of ligand clusters, the receptor-ligand binding constant can be significantly enhanced due to the less translational entropy loss of lipid-raft domains in the model cell membranes upon the formation of additional complexes. For ligands randomly or uniformly immobilized on the substrate, the binding constant is rather decreased since the receptors localized in lipid-raft domains have to pay an energetic penalty in order to bind ligands. Our findings help to understand why cell-substrate adhesion experiments for measuring the impact of lipid rafts on the receptor-ligand interactions led to contradictory results.
\end{abstract}

DOI: 10.1103/PhysRevE.97.012405

\section{INTRODUCTION}

The adhesion of cells to cells, tissues, and extracellular matrix governs numerous biological processes such as signal transduction, tissue formation, immune responses, and cell locomotion [1-3]. In cell-cell adhesion, both binding partners are mobile, whereas in cell-extracellular matrix and cell-tissue adhesion, mobile receptors on the cell membranes often bind immobile ligand proteins presented on the apposing surface. The adhesion is caused by the specific binding of receptors and ligands anchored on the apposing surfaces [4,5], which is typically quantified by the two-dimensional binding equilibrium constant [6-10],

$$
K=\frac{[R L]}{[R][L]},
$$

with $[R],[L]$, and $[R L]$ the area concentrations of free receptors, free ligands, and receptor-ligand complexes. A variety of experimental techniques including fluorescence spectroscopy [11-14], micropipette aspiration [15-19], atomic force microscopy [20-22], and the flow chamber [23-25] have been used to characterize the binding constant, leading to values that differ by orders of magnitude [26,27]. Computer simulations

\footnotetext{
*hujinglei@nju.edu.cn

${ }^{\dagger}$ songf@lnm.imech.ac.cn
}

have recently shown that membrane shape fluctuations [8-10] and localization of proteins in lipid rafts (cholesterol- and sphingolipid-rich membrane domains) [28] significantly affect the binding constant of the anchored proteins. The ligands are immobilized on surfaces in micropipette aspiration [15-19] and flow chamber [23-25] measurements, whereas in the other studies ligands are anchored to membranes and diffuse laterally along the membrane surfaces. A natural question arises of whether the receptors bind more strongly or weakly to mobile ligands than immobile ones.

Cumulative evidence indicates that cell membranes are not structurally homogeneous, but rather consist of lipid rafts, nanosized membrane domains enriched in cholesterol and saturated phospholipids [29-33]. The raft domains can lead to a heterogeneous distribution of proteins in the membranes by recruiting them to variable extents and function as signaling platforms to facilitate protein-protein cis- interactions on a single membrane [34]. For the trans- interactions, however, experiments of cell-substrate adhesion with immobile ligands have led to contradictory results regarding the effect of lipid rafts on receptor-ligand binding [35-40]. For example, Wang et al. [37] reported that lipid rafts colocalize the integrin $\beta 1$ and enhance their binding to fibronectin. Norman et al. [38] found that the disruption of lipid rafts by depleting cholesterol with methyl- $\beta$-cyclodextrin $(\mathrm{M} \beta \mathrm{CD})$ increases the cell-substrate detachment. Conversely, Murai et al. [39] showed that the localization of CD44 in lipid rafts inhibits the CD44-hyaluronan interactions and exerts negative control on the $\mathrm{T}$ cell adhesion 


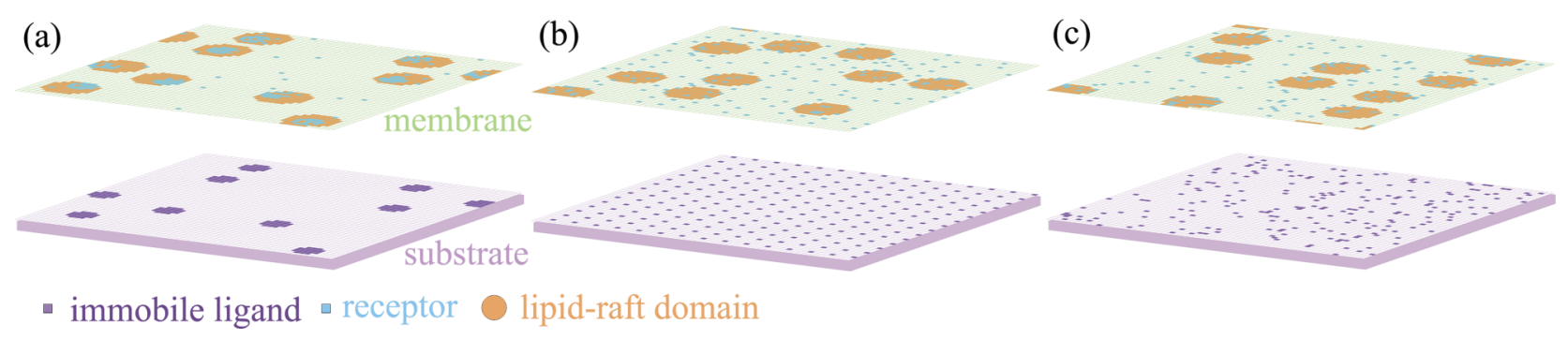

FIG. 1. Simulation snapshots for adhesion of the cell membrane with lipid-raft domains (orange) and receptors (light blue) to a flat rigid substrate with ligands (purple) via specific receptor-ligand binding. The ligands are immobilized (a) in the form of clusters, (b) uniformly, or (c) randomly on the substrate. The cell membrane and substrate are modeled by two-dimensional square lattices. Each receptor or ligand occupies a single square patch. A receptor binds to a ligand if they are apposing each other and within the interaction range of binding potential given in Eq. (2). Lipid rafts in the cell membrane are represented by clusters of adjacent patches, and diffuse laterally along the membrane surface. The mobile receptors prefer raft domains to the non-raft region of the membrane.

to hyaluronan-coated substrate. Evani and Ramasubramanian [40] observed that the adhesion of infected monocytes to the microchannels coated with E-selectin is enhanced due to the disruption of lipid rafts. The change in cell-substrate adhesion strength reflects a possible variation in both the receptorligand complex number and binding affinity in the adhesion zone.

Here we report Monte Carlo (MC) simulations of a mesoscopic model for cell-substrate adhesion. We systematically varied the area fraction and size of lipid-raft domains in the cell membranes, and the affinity of rafts to the anchored receptors, and considered three types of ligand distribution on the substrate. Remarkably different from the positive role of lipid rafts in facilitating receptor-ligand interactions in cell-cell adhesion seen in our previous work [28], the effect of these microdomains on the binding constant of membrane-anchored receptors and substrate-immobilized ligands depends strongly on the ligand distribution. For ligands immobilized in the form of clusters on the substrate as shown in Fig. 1(a), their binding with the receptors localized in the raft domains is enhanced because of the less translational entropy loss of the rafts upon receptor-ligand complex formation, yielding an increase of one order of magnitude in the binding constant; for ligands uniformly or randomly immobilized on the substrate as shown in Figs. 1(b) and 1(c), the binding constant is found to be smaller than that for mobile ligands anchored to supported, planar membranes, since the receptors localized in the lipid rafts have to pay an energetic penalty in order to bind immobile ligands.

The rest of the manuscript is structured as follows. Section II outlines the model for cell-substrate adhesion and the MC simulation method as well as the physical parameters chosen according to experiments. Our main simulation results are presented in Sec. III, and Sec. IV summarizes our findings. For detailed comparison, additional data are presented in Figs. 6-10 in the Appendix.

\section{MODEL AND METHOD}

In our model of cell-substrate adhesion, both the cell membrane and rigid planar substrate are represented by twodimensional square lattices [28,41-43] as shown in Fig. 1. Each receptor or ligand occupies a single square patch of size $a$. The adhesion energy from the specific binding of receptors and ligands on the two surfaces assumes

$$
\mathcal{H}_{\mathrm{ad}}=\sum_{i}-\delta_{i} U_{\mathrm{b}} \theta\left(l_{\mathrm{b}} / 2-\left|l_{i}-l_{\mathrm{c}}\right|\right),
$$

where the square-well binding potential has the depth $U_{\mathrm{b}}$ and width $l_{\mathrm{b}} . l_{\mathrm{c}}$ is the average length of the receptor-ligand complex, and $l_{i}$ the local separation of the two surfaces at lattice site $i$. $\delta_{i}$ is 1 if the two patches at lattice site $i$ are occupied by receptor and ligand proteins, respectively, and otherwise 0 . The Heaviside step function $\theta(x)$ implies that a complex cannot be formed if the two proteins are separated beyond the binding range. The square-well potential in Eq. (2) mimics the specific binding interactions of rigid proteins. For proteins with flexible segments, their conformational entropy might have a significant impact on binding and should be taken into account via detailed modeling, e.g., the bead-spring model of polymer chains. This interesting aspect goes beyond the scope of the present work and should be addressed in future studies.

Each lipid raft is described by a cluster of adjacent square patches, and diffuses freely along the membrane surface. Since the raft domains are dynamically floating in the membranes and do not coalesce over a certain period of time [44] (e.g., due to the stabilization of the local membrane curvature [45]), we impose hard-core repulsions between any two rafts. This also allows us to study how the raft size plays a role in cell-substrate adhesion. To account for the preferential partitioning of receptors in lipid-raft domains [46], we introduce the energy gain $\Delta U$ for a receptor to move from the nonraft region of the membrane to a raft domain, i.e., the raft affinity to receptors. Unlike the mobile receptors, the ligands are immobilized on the substrate. For receptors anchored to flexible membranes, one has the additional Helfrich bending energy,

$$
\mathcal{H}_{\text {be }}=\frac{\kappa}{2 a^{2}} \sum_{i}\left(\Delta_{\mathrm{d}} l_{i}\right)^{2},
$$

which governs the elastic deformation of the membrane. $\kappa$ is the membrane bending rigidity and $\Delta_{\mathrm{d}} l_{i}$ the discretized 

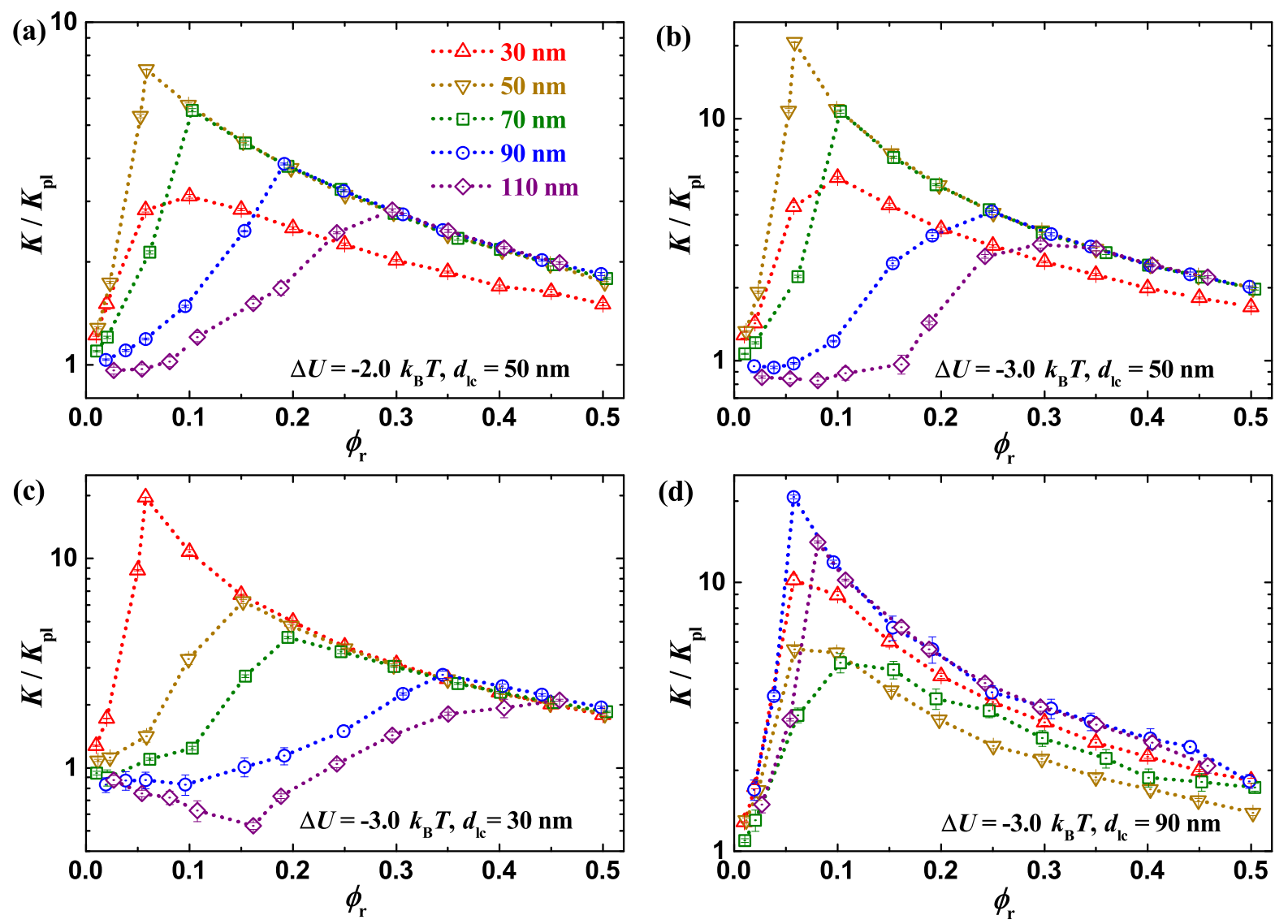

FIG. 2. Binding equilibrium constant $K$ of receptors anchored to planar membranes and ligands immobilized in clusters on the apposing substrate as in Fig. 1(a). The simulation data points are from systems with ligand clusters of different diameter $d_{\mathrm{lc}}$ and lipid rafts of varying area fraction $\phi_{\mathrm{r}}$, diameter $d_{\mathrm{r}}$, and affinity $\Delta U$ to receptors. Each data point is the statistical average over 20 independent runs with random distribution of ligand clusters on the substrate. The overall concentrations of the receptors and ligands are $[R]_{0} \approx 560 \mu \mathrm{m}^{-2},[L]_{0} \approx 580 \mu \mathrm{m}^{-2}$. $K_{\mathrm{pl}} \equiv a^{2} e^{U_{\mathrm{b}} / k_{\mathrm{B}} T}$ is the receptor-ligand binding constant in the absence of lipid rafts, independent of the spatial distribution of immobile ligands on the substrate. The dotted lines are only a guide for the eye. Note the logarithmic scale of the vertical axes.

Laplacian of the local membrane-substrate separation $l_{i}$ at lattice site $i[4,42,43]$.

We employ the standard Metropolis MC method to simulate the membrane-substrate adhesion systems. There are three types of MC trial moves including the lateral movement of receptors and raft domains, and vertical displacement of the membrane patches in the case of a flexible membrane. The proportion of those trial moves in each MC step is chosen according to the physical time scales. For more details, see Ref. [28]. We emphasize that the exact values for diffusion coefficients of the proteins and raft domains do not alter the binding constant $K$, since $K$ is an equilibrium quantity independent of the dynamic properties of the system.

We have simulated the adhering membrane with an area of $600 \times 600 \mathrm{~nm}^{2}$ and periodic boundary conditions. For the square-well potential, we take binding energy $U_{\mathrm{b}}=5 k_{\mathrm{B}} T$, potential range $l_{\mathrm{b}}=1 \mathrm{~nm}$, and complex length $l_{c}=15 \mathrm{~nm}$ [41,42]. We choose typical values of several hundred molecules per square micrometer for the overall receptor and ligand concentrations [42]. The raft domains have a diameter ranging from 30 to $100 \mathrm{~nm}$ [47]. The raft affinity $\Delta U=-2.0 k_{\mathrm{B}} T$ and $-3.0 k_{\mathrm{B}} T$ are adopted so that the protein concentration in lipid rafts is within the experimentally reported range of around $10^{3}-10^{4} \mu \mathrm{m}^{-2}$ [48]. Considering the variation of raft concentration in the membrane contact zone accompanying the intercellular interaction process [46], we vary the area fraction of the raft domains up to $50 \%$ of the membrane surface area $[49,50]$. Additionally, the bending rigidity of lipid membranes $\kappa$ is typically $10 k_{\mathrm{B}} T$ [8]. We choose $\kappa=10 k_{\mathrm{B}} T$ for the non-raft membrane patches, and $10 k_{\mathrm{B}} T$ or $20 k_{\mathrm{B}} T$ for the raft domains in the case of flexible membranes [51]. In our simulations, we calculate the binding constant $K$ from the area concentrations according to Eq. (1). For ligands immobilized in the form of clusters or immobilized randomly on the substrate, the simulation results are the statistical average over 20 independent realizations.

\section{RESULTS AND DISCUSSION}

\section{A. Receptors anchored to planar membranes}

Localization of receptors in lipid rafts affects their binding to ligands immobilized on the planar substrate. Figure 2 shows 

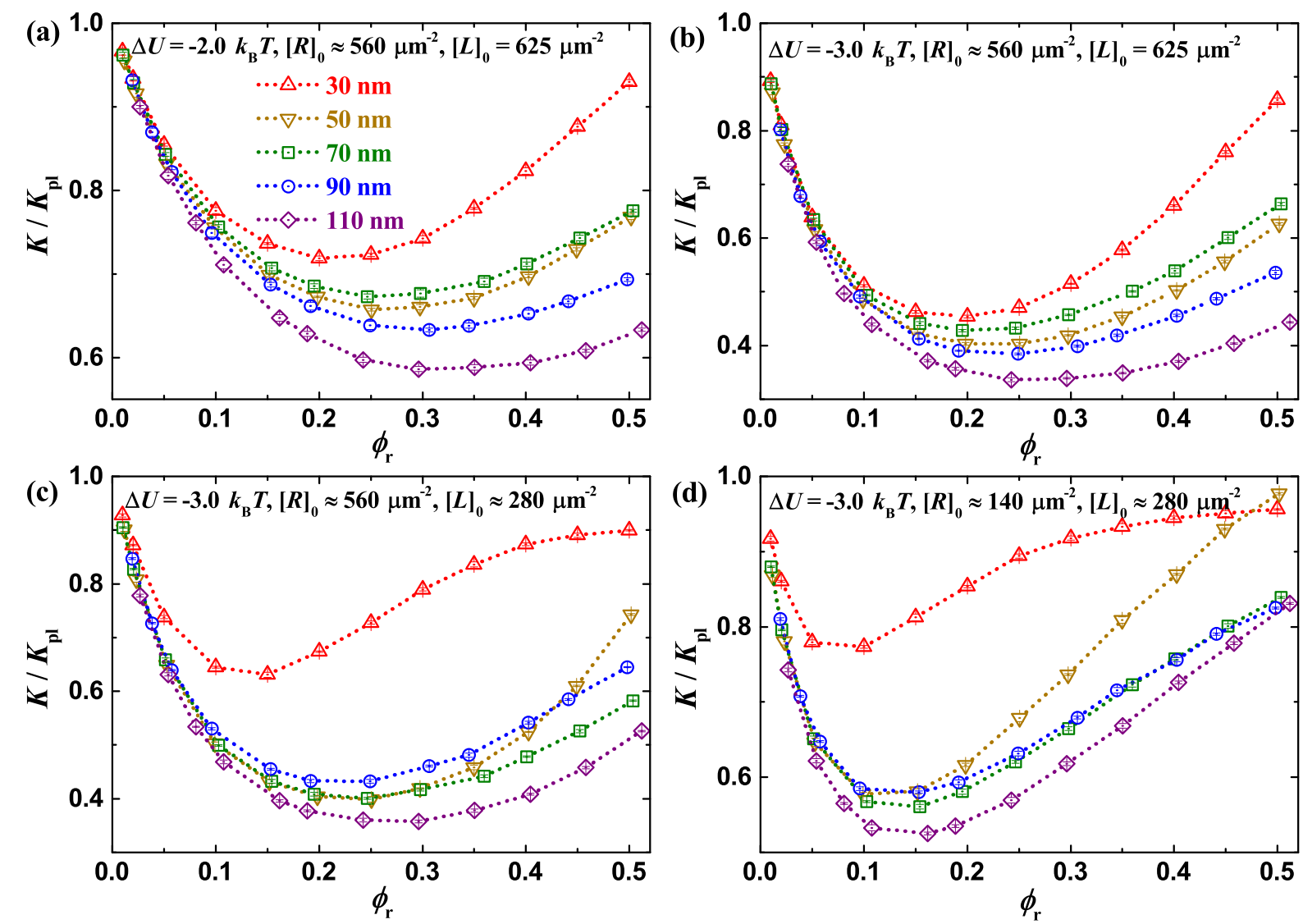

FIG. 3. Binding equilibrium constant $K$ of receptors anchored to planar membranes and ligands immobilized uniformly on the apposing substrate as in Fig. 1(b). The meaning of all symbols is the same as in Fig. 2.

the measured binding constant $K$ rescaled by $K_{\mathrm{pl}}$ as a function of area fraction $\phi_{\mathrm{r}}$ of lipid rafts with different size and affinity to receptors. Here, $K_{\mathrm{pl}} \equiv a^{2} e^{U_{\mathrm{b}} / k_{\mathrm{B}} T}$ is the receptor-ligand binding constant in the absence of lipid rafts, independent of the spatial distribution of immobile ligands on the substrate. $K_{\mathrm{pl}}$ also denotes the binding constant of the same but mobile receptor and ligand proteins anchored to two apposing planar membranes without shape fluctuations, irrespective of whether the membranes are homogeneous or with lipid-raft domains. Given the raft size $d_{\mathrm{r}}$ and affinity $\Delta U$ to receptors, as well as ligand cluster size $d_{\mathrm{lc}}$, the binding constant $K$ varies with the raft area fraction $\phi_{\mathrm{r}}$, and there exists a maximum even much greater than $K_{\mathrm{pl}}$.

Such enhancement of binding can be understood from the translational entropy of lipid-raft domains in the cell membrane. The binding of a first receptor $\left(R_{1}\right)$ localized in a raft of area $A$ to an immobile ligand $\left(L_{1}\right)$ causes a translational entropy loss $\Delta S_{0} \approx k_{\mathrm{B}} \ln \left(A / A_{\mathrm{me}}\right)+k_{\mathrm{B}} \ln \left(a^{2} / A\right)$. Here, $A_{\mathrm{me}}$ and $A$ represent the translational phase space volume of the raft before and after the binding; $a^{2}$ and $A$ are the corresponding terms for the receptor $R_{1}$. Given the complex $R_{1}-L_{1}$, a second receptor $\left(R_{2}\right)$ in the same raft binds to another ligand $\left(L_{2}\right)$ with a translational entropy loss $\Delta S_{1} \approx k_{\mathrm{B}} \ln \left(A_{1} / A\right)+k_{\mathrm{B}} \ln \left(a^{2} / A\right)$, where the area $A_{1}$ available for raft translation under the constraint of complexes $R_{1}-L_{1}$ and $R_{2}-L_{2}$ depends on the distance between $L_{1}$ and $L_{2}$ (see Fig. 6 for the dependence of binding constant $K$ on the ligand distance). For the case of a ligand cluster as depicted in Fig. 1(a), one can attain $A_{1} \approx A$ and then $\Delta S_{0}<\Delta S_{1}$ since $A$ is much smaller than the membrane area $A_{\text {me }}$. The less translational entropy loss of lipid rafts therefore facilitates the formation of additional complexes. The translational entropy consideration explains the increase of apparent binding constant $K$ with the area fraction $\phi_{\mathrm{r}}$ of the rafts for a small value of $\phi_{\mathrm{r}}$, and would generally apply to rafts of noncircular shapes. After reaching the maximum, $K$ decreases with $\phi_{\mathrm{r}}$ since the local receptor concentration in each raft reduces down to the overall initial concentration $[R]_{0}$. Comparison of Figs. 2(a) and 2(b) suggests that large raft affinity leads to strong binding of the receptors to immobile ligands, and the ratio of maximal binding constant $K$ for each raft size at $\Delta U=-2 k_{\mathrm{B}} T$ and $-3 k_{\mathrm{B}} T$ is around $e^{1} \approx 2.8$. It is interesting to point out that the greatest maximal $K$ occurs for the raft with the same size as the ligand cluster, irrespective of the raft affinity and overall concentrations of receptors and ligands as can be seen in Figs. 2 and 7.

In addition to the enhancement, preferential partitioning in rafts might also hinder the binding of receptors to immobile ligands. As shown in Figs. 2(a)-2(c), the binding constant $K$ can be less than $K_{\mathrm{pl}}$ for a small area fraction of relatively large rafts. The decrease in $K$ results from the excessive free receptors in a raft that need to overcome raft affinity in order 

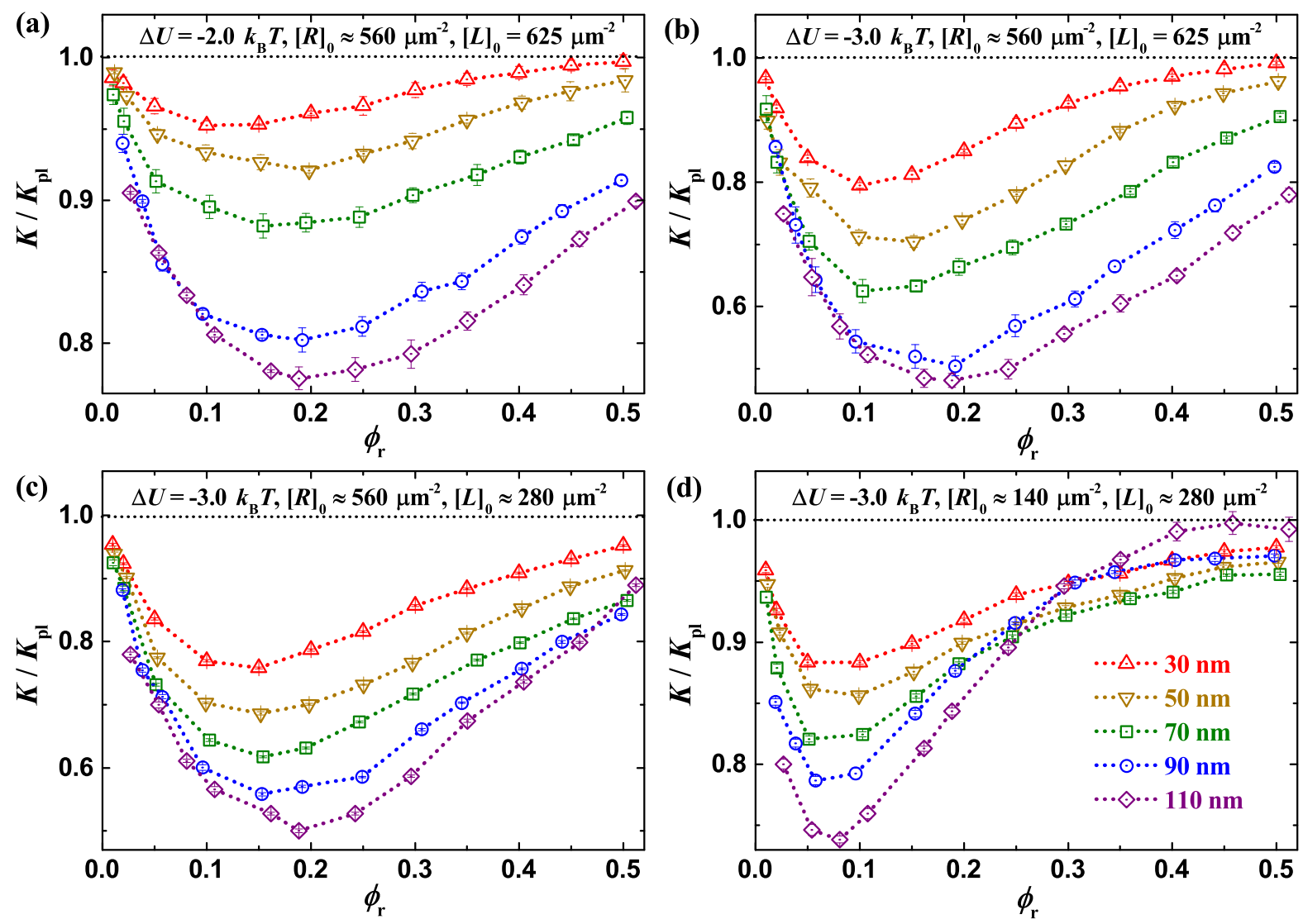

FIG. 4. Binding equilibrium constant $K$ of receptors anchored to planar membranes and ligands immobilized randomly on the apposing substrate as in Fig. 1(c). The data points are the statistical average over 20 independent runs with random distribution of ligands. The meaning of all symbols is the same as in Fig. 2.

to bind with the immobilized unbound ligands outside of the region apposing the raft on the substrate; see the number of excessive receptors as a function of raft area fraction in Fig. 8 corresponding to the diamond data points in Fig. 2(c). A close inspection of Figs. 2 and 7 reveals that strong raft affinity, large size of raft relative to ligand cluster, and large ratio of overall concentrations $[R]_{0} /[L]_{0}$ lead to large excess of receptors and therefore small $K$.

We now turn to the binding of mobile receptors to ligands uniformly or randomly immobilized on the substrate as illustrated in Figs. 1(b) and 1(c). Figures 3 and 4 show that the binding constant $K$ measured for both distributions of ligands is less than $K_{\mathrm{pl}}$ over the wide range of raft area fraction $\phi_{\mathrm{r}}$ for different overall concentrations of receptors and ligands. Smaller raft affinity $\Delta U$ and concentration ratio $[R]_{0} /[L]_{0}$ yield less excess of receptors localized in rafts and thus greater binding constant $K$. Comparison of Figs. 3 and 4 indicates that the receptors bind more strongly to randomly distributed ligands than to uniformly distributed ones. This can be attributed to local aggregation of randomly immobile ligands that requires less translational entropy loss of the rafts upon receptor-ligand binding as discussed in the previous case of ligand clusters. The results in Fig. 4 for random distribution of immobile ligands on the substrate are a statistical average over 20 independent realizations. For individual realizations, we do observe $K>K_{\mathrm{pl}}$ at $[R]_{0} \approx 140 \mu \mathrm{m}^{-2},[L]_{0} \approx 280 \mu \mathrm{m}^{-2}$, $\phi_{\mathrm{r}}>0.4$, and $d_{\mathrm{r}}=110 \mathrm{~nm}$.

\section{B. Receptors anchored to flexible cell membranes}

The membranes in cell-substrate adhesion are often subjected to thermal excitations and undergo shape fluctuations. We consider the binding of receptors anchored to flexible membranes and ligands immobilized in the form of clusters, uniformly, or randomly on apposing substrates. The measured binding constants in Figs. 5(a)-5(c) show similar dependence on the raft area fraction $\phi_{\mathrm{r}}$ as for planar membranes without shape fluctuations, but the numerical values are considerably smaller since the thermally excited shape fluctuations of flexible membranes disfavor the formation of receptor-ligand complexes. In the case of ligand clusters, the binding constant $K$ can exceed $K_{\mathrm{pl}}$ for flexible membranes, implying that the enhancement due to translational entropy of rafts overweighs the suppression from shape fluctuations of the membranes. For uniform distribution of ligands on the substrate, both excess of receptors in rafts and membrane shape fluctuations lead to the binding constant $K$ being smaller than the constant $K_{0}$ for flexible homogeneous membranes without lipid-raft domains; see Figs. 5(b) and $9\left(\Delta U=-3.0 k_{\mathrm{B}} T,[R]_{0} \approx 140 \mu \mathrm{m}^{-2}\right.$, $[L]_{0}=625 \mu \mathrm{m}^{-2}$ ). Figures 5(c) and 5(d) illustrate a delicate 

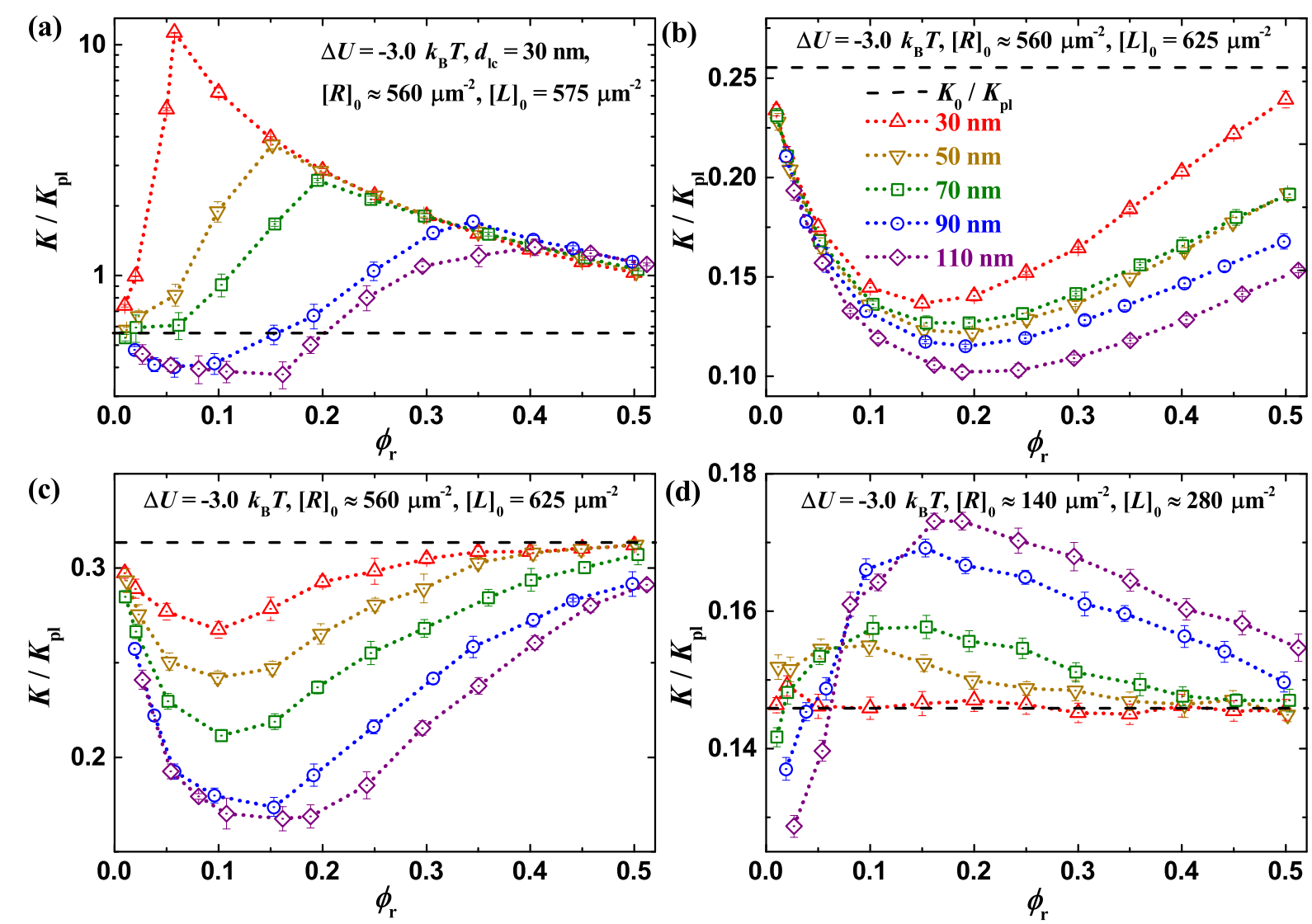

FIG. 5. Binding equilibrium constant $K$ of receptors anchored to flexible membranes and ligands immobilized on the substrate apposing the membranes. The bending rigidity of the membranes is chosen to be $\kappa=10 k_{\mathrm{B}} T$. The ligands are immobilized (a) in the form of clusters, (b) uniformly, or (c,d) randomly on the substrate. The horizontal dashed lines indicate the receptor-ligand binding constant $K_{0}$ for the flexible membranes without lipid-raft domains. The meaning of all symbols is the same as in Fig. 2.

interplay of receptor excess and membrane configurational entropy for random distribution of ligands. At large overall concentrations, the receptor excess dominates and $K<K_{0}$; at small overall concentrations, the receptor-ligand complexes are mostly formed within or near the rafts apposing the substrate region with aggregation of randomly distributed ligands, favoring the configurational entropy of the membrane.

Lipid-raft domains are often more rigid than the non-raft regions of the membranes [51]. We have also simulated membranes with raft domains of bending rigidity $20 k_{\mathrm{B}} T$ and nonraft patches $10 k_{\mathrm{B}} T$, and obtained in Fig. 10 similar dependence of binding constant $K$ on raft area fraction $\phi_{\mathrm{r}}$ as in Fig. 5 for membranes without bending rigidity contrast. This bending rigidity contrast leads to slightly enhanced binding of the immobile ligands to the receptors anchored to fluctuating membranes for the three types of ligand distribution, since the rigid raft domains have less conformational entropy to lose upon the binding of ligands to the receptors localized in the rafts.

\section{CONCLUSION AND OUTLOOK}

We have investigated the impact of lipid rafts on the binding of membrane-anchored receptors and substrate-immobilized ligands by using Monte Carlo simulations of adhesion of multicomponent membranes to a flat substrate. We find that the binding constant of membrane-anchored receptors and substrate-immobilized ligands can be enhanced or reduced by the presence of lipid-raft domains in the cell membrane, dependent on the distribution of immobile ligands on the substrate. For ligands immobilized in the form of clusters, the binding constant can even be one order of magnitude larger than that of the same but mobile receptors and ligands for two planar, supported membranes without thermal shape fluctuations as shown in Fig. 2. Such enhancement is attributed to the translational entropy of lipid-raft domains localizing the receptors, and indicates a cooperative binding different from that for receptors and ligands anchored to elastic homogeneous membranes without rafts as in Ref. [9], which originates from shape fluctuations of the membranes. Interestingly, the greatest maximal value of binding constant occurs when the raft domains match the size of the ligand cluster.

In addition to the enhancement, our results in Figs. 3 and 4 for ligands immobilized uniformly or randomly on the substrate reveal that lipid-raft domains might also function as a negative regulator of binding since the excessive free receptors localized in raft domains have to overcome the raft affinity in order to bind the unbound substrate-immobilized ligands outside of the region apposing the raft. We anticipate that the aggregation of receptors due to other mechanisms such as protein-protein $c i s$ - attraction [52,53] could also lead to 


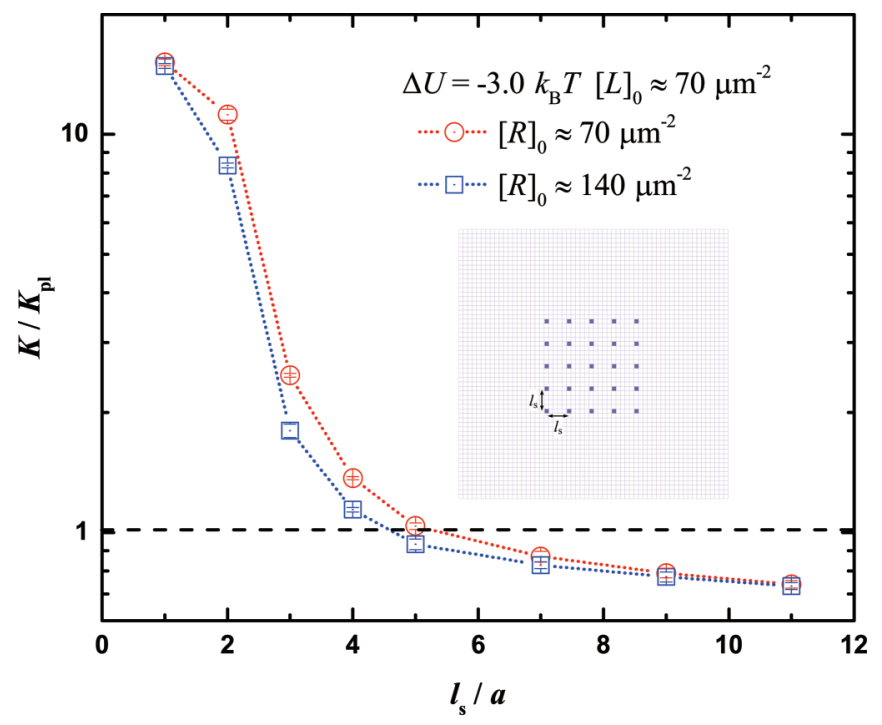

FIG. 6. Binding equilibrium constant $K$ of receptors anchored to planar cell membranes and ligands immobilized on the apposing substrate as a function of ligand separation $l_{\mathrm{s}}$. The cell membrane consists of one single lipid-raft domain with diameter $d_{\mathrm{r}}=110 \mathrm{~nm}$. The horizontal dashed line indicates the binding constant $K$ for mobile ligands.

receptor excess and therefore impact the binding to immobile ligands.

The results in Figs. 5 and 10 for flexible adhering membranes are qualitatively similar to those of planar membranes without thermal fluctuations. For ligands randomly immobilized on the substrate, the binding can be stronger than in the case of homogeneous membranes without lipid-raft domains as shown in Fig. 5(d), indicating the interplay between membrane conformational entropy and the receptor excess in lipid-raft domains.

Our findings help to explain the seemingly contradictory results regarding the role of lipid rafts in the binding of membrane-anchored receptors and substrate-immobilized ligands in cell-substrate adhesion experiments [35-40], where the distribution of immobile ligands on the substrate should be carefully taken into account. We provided straightforward physical arguments as confirmed by the simulation data to corroborate our findings. Together with previous work on the impact of lipid rafts on the receptor-ligand binding in cell-cell adhesion [28], our studies suggest that lipid rafts might function differently as required in cell-cell adhesion and the adhesion of cells to tissues and extracellular matrix under physiological conditions.

Our statistical model does not take into account the complex cytoskeletal network that anchors to the cell membranes. As pointed out in Ref. [42], the anchoring to cytoskeleton might suppress membrane fluctuations on length scales larger than the mesh size of the network [54,55], whereas active cell processes involved in the cytoskeleton might enhance the fluctuations [56,57]. Both are likely to perturb the equilibrium situation. However, our results still apply for membrane regions with local equilibrium of receptor-ligand binding.

\section{ACKNOWLEDGMENTS}

L.L. thanks Mian Long and Shouqin Lü for insightful discussions. This work was supported by the Programs in the National Natural Science Foundation of China (Grants No. 11472285, No. 11232013, No. 21504038 and No. 11402193), the Strategic Priority Research Program of the Chinese Academy of Sciences (Grant No. XDB22040102), and the National Key Research and Development Program of China (Grant No. 2016YFA0501601). J.H. also acknowledges the financial support by Fundamental Research Funds for the Central Universities and Shenzhen Science and Technology Innovation Committee (Grant No. JCYJ20160531151105346).

\section{APPENDIX}

We provide here additional simulation results in Figs. 6-10 for detailed comparison with the results in the main text.
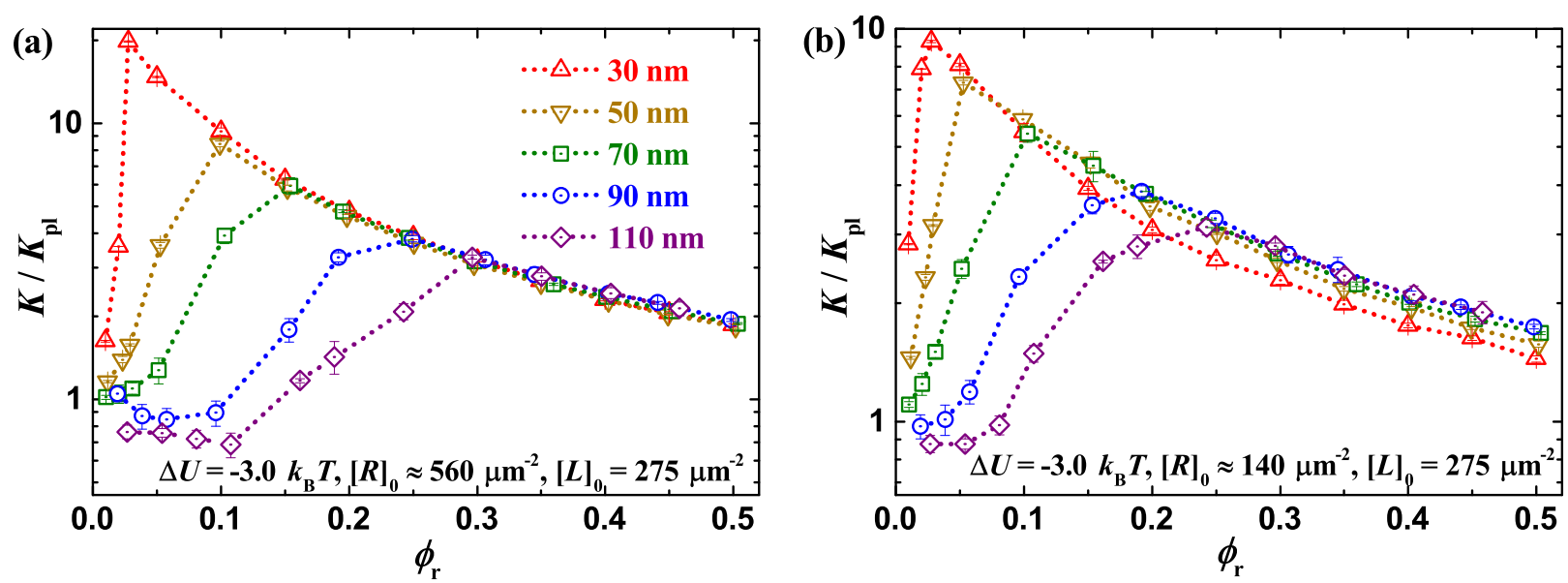

FIG. 7. Binding constant $K$ of the same receptors and ligands as in Fig. 2 obtained from simulations with ligand clusters of diameter $d_{\mathrm{lc}}=30 \mathrm{~nm}$ and different overall concentrations of the receptors and ligands. 


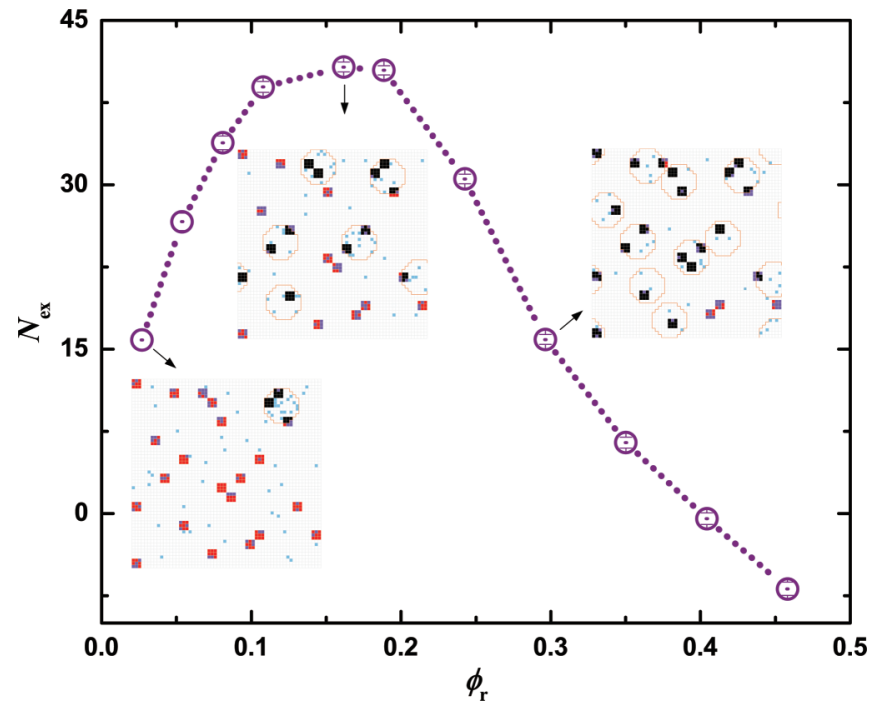

FIG. 8. The number of unbound receptors in lipid rafts that exceeds the number of immobilized unbound ligands apposing raft domains $N_{\mathrm{ex}}$ as a function of area fraction $\phi_{\mathrm{r}}$ of lipid rafts with fixed size $d_{\mathrm{r}}=110 \mathrm{~nm}$ and affinity to receptors $\Delta U=-3.0 k_{\mathrm{B}} T$ corresponding to the purple data in Fig. 2(c). The cluster size of immobilized ligands is $d_{\mathrm{lc}}=30 \mathrm{~nm}$. In the inserted top-view simulation snapshots for different $\phi_{\mathrm{r}}$, the orange lines enclose the raft domains on the cell membranes. The light blue and purple patches are with free receptors and ligands, the black with bound receptors and ligands in membrane raft domains, and the red with the rest of the receptor-ligand complexes.

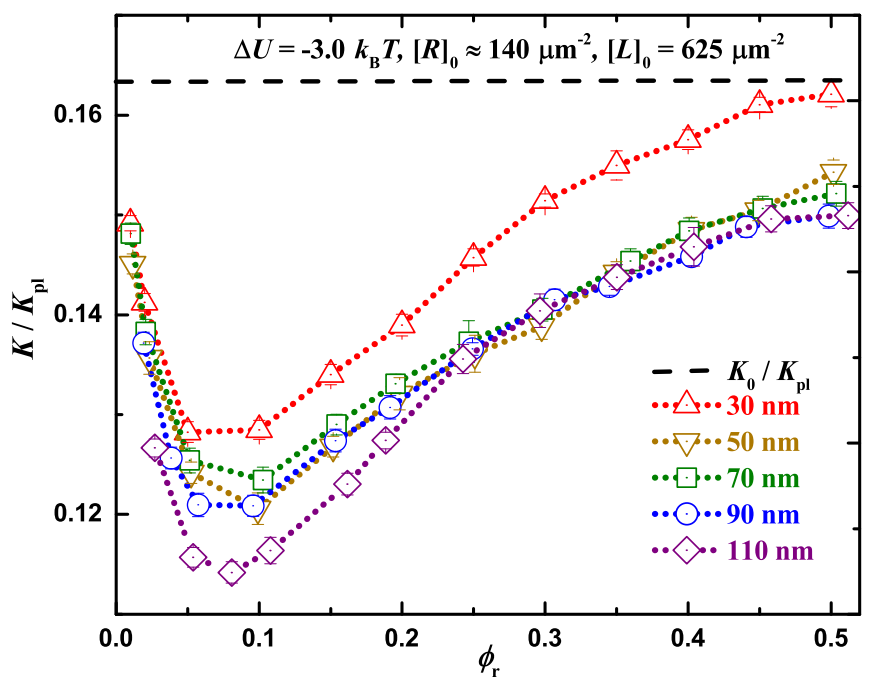

FIG. 9. Binding equilibrium constant $K$ of receptors anchored to flexible cell membranes and ligands immobilized uniformly on the substrate apposing the membranes. The horizontal dashed line indicates the receptor-ligand binding constant $K_{0}$ for the flexible membranes without lipid rafts. The meaning of all symbols is the same as in Fig. 2.
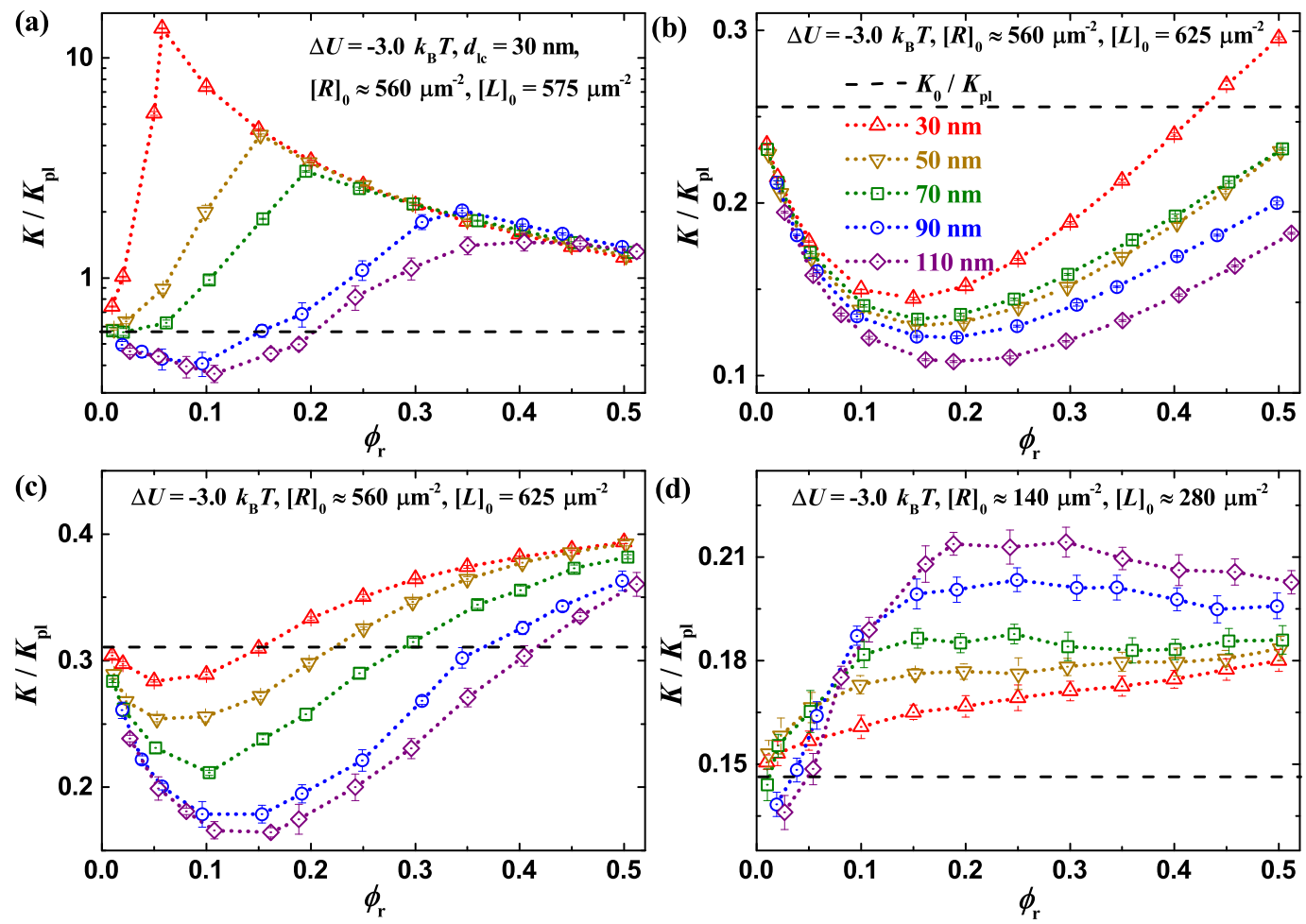

FIG. 10. Binding equilibrium constant $K$ of receptors anchored to flexible membranes and ligands immobilized on the substrate apposing the membranes. The raft domains have a bending rigidity of $20 k_{\mathrm{B}} T$ and non-raft patches $10 k_{\mathrm{B}} T$ in the membranes. The horizontal dashed lines indicate the receptor-ligand binding constant $K_{0}$ for the flexible membranes without lipid-raft domains. The meaning of all symbols is the same as in Fig. 2. 
[1] P. A. van der Merwe and O. Dushek, Nat. Rev. Immunol. 11, 47 (2011).

[2] B. Alberts, A. Johnson, J. Lewis, M. Raff, K. Roberts, and P. Walter, Molecular Biology of the Cell (Garland Science, New York, 2014).

[3] K. D. Sumigray and T. Lechler, Curr. Top. Dev. Biol. 112, 383 (2015).

[4] T. R. Weikl, M. Asfaw, H. Krobath, B. Różycki, and R. Lipowsky, Soft Matter 5, 3213 (2009).

[5] G. K. Xu, Z. Liu, X. Q. Feng, and H. Gao, Phys. Rev. E 93, 032411 (2016).

[6] J. B. Huppa, M. Axmann, M. A. Mörtelmaier, B. F. Lillemeier, E. W. Newell, M. Brameshuber, L. O. Klein, G. J. Schütz, and M. M. Davis, Nature 463, 963 (2011).

[7] Y. Wu, J. Vendome, L. Shapiro, A. Ben-Shaul, and B. Honig, Nature 475, 510 (2011).

[8] G. K. Xu, J. Hu, R. Lipowsky, and T. R. Weikl, J. Chem. Phys. 143, 243136 (2015).

[9] J. Hu, R. Lipowsky, and T. R. Weikl, Proc. Natl. Acad. Sci. USA 110, 15283 (2013).

[10] J. Hu, G. K. Xu, R. Lipowsky, and T. R. Weikl, J. Chem. Phys. 143, 243137 (2015).

[11] D. M. Zhu, M. L. Dustin, C. W. Cairo, and D. E. Golan, Biophys. J. 92, 1022 (2007).

[12] T. P. Tolentino, J. Wu, V. I. Zarnitsyna, Y. Fang, M. L. Dustin, and C. Zhu, Biophys. J. 95, 920 (2008).

[13] G. P. O’Donoghue, R. M. Pielak, A. A. Smoligovets, J. J. Lin, and J. T. Groves, eLife 2, e00778 (2013).

[14] G. Mocz and J. A. Ross, Methods Mol. Biol. 1008, 169 (2013).

[15] W. Chen, E. A. Evans, R. P. McEver, and C. Zhu, Biophys. J. 94, 694 (2008).

[16] J. Huang, V. I. Zarnitsyna, B. Y. Liu, L. J. Edwards, N. Jiang, B. D. Evavold, and C. Zhu, Nature 464, 932 (2010).

[17] B. Y. Liu, W. Chen, B. D. Evavold, and C. Zhu, Cell 157, 357 (2014).

[18] B. Y. Liu, S. Zhong, K. Malecek, L. A. Johnson, S. A. Rosenberg, C. Zhu, and M. Krogsgaard, Eur. J. Immunol. 44, 239 (2014).

[19] E. B. Lomakina and R. E. Waugh, Biophys. J. 86, 1223 (2004).

[20] M. Long, S. Q. Lü, and G. Y. Sun, Cell. Mol. Immunol. 3, 79 (2006).

[21] T. L. L. Doan, Y. Guerardel, P. Loubiere, M. Mercier-Bonin, and E. Dague, Biophys. J. 101, 2843 (2011).

[22] M. M. Zhang, B. Wang, and B. Q. Xu, J. Phys. Chem. B 118, 6714 (2014).

[23] C. D. Paschall, W. H. Guilford, and M. B. Lawrence, Biophys. J. 94, 1034 (2008).

[24] T. Yago, V. I. Zarnitsyna, A. G. Klopocki, R. P. McEver, and C. Zhu, Biophys. J. 92, 330 (2007).

[25] L. Limozin, P. Bongrand, and P. Robert, Sci. Rep. 6, 35193 (2016).

[26] M. L. Dustin, S. K. Bromley, M. M. Davis, and C. Zhu, Annu. Rev. Cell Dev. Biol. 17, 133 (2001).

[27] C. Zhu, N. Jiang, J. Huang, V. I. Zarnitsyna, and B. D. Evavold, Immunol. Rev. 251, 49 (2013).
[28] L. Li, J. Hu, X. H. Shi, Y. F. Shao, and F. Song, Soft Matter 13, 4294 (2017).

[29] D. Lingwood and K. Simons, Science 327, 46 (2010).

[30] K. Simons and M. J. Gerl, Nat. Rev. Mol. Cell Biol. 11, 688 (2010).

[31] U. E. Martinez-Outschoorn, F. Sotgia, and M. P. Lisanti, Nat. Rev. Cancer 15, 225 (2015).

[32] W. Wu, X. S. Shi, and C. Q. Xu, Nat. Rev. Immunol. 16, 690 (2016).

[33] E. Sezgin, I. Levental, S. Mayor, and C. Eggeling, Nat. Rev. Mol. Cell Biol. 18, 361 (2017).

[34] F. Mollinedo and C. Gajate, Adv. Biol. Regul. 57, 130 (2015).

[35] Q. Huang, H. M. Shen, G. H. Shui, M. R. Wenk, and C. N. Ong, Cancer Res. 66, 5807 (2006).

[36] O. G. Ramprasad, G. Srinivas, K. S. Rao, P. Joshi, J. P. Thiery, S. Dufour, and G. Pande, Cell Motil. Cytoskeleton 64, 199 (2007).

[37] C. R. Wang, Y. Yoo, H. P. Fan, E. Kim, K. L. Guan, and J. L. Guan, J. Biol. Chem. 285, 29398 (2010).

[38] L. L. Norman, R. J. Oetama, M. Dembo, F. Byfield, D. A. Hammer, I. Levitan, and H. Aranda-Espinoza, Cell. Mol. Bioeng. 3, 151 (2010).

[39] T. Murai, C. Sato, M. Sato, H. Nishiyama, M. Suga, K. Mio, and H. Kawashima, J. Cell Sci. 126, 3284 (2013).

[40] S. J. Evani and A. K. Ramasubramanian, Sci. Rep. 6, 12 (2016).

[41] T. R. Weikl and R. Lipowsky, Biophys. J. 87, 3665 (2004).

[42] H. Krobath, B. Różycki, R. Lipowsky, and T. R. Weikl, Soft Matter 5, 3354 (2009).

[43] H. Krobath, B. Różycki, R. Lipowsky, and T. R. Weikl, PLoS One 6, e23284 (2011).

[44] A. Pralle, P. Keller, E.-L. Florin, K. Simons, and J. K. H. Hörber, J. Cell Biol. 148, 997 (2000).

[45] B. Różycki, T. R. Weikl, and R. Lipowsky, Phys. Rev. Lett. 100, 098103 (2008).

[46] S. K. Pierce, Nat. Rev. Immunol. 2, 96 (2002).

[47] H. Kalthoff, Death Receptors and Cognate Ligands in Cancer (Springer-Verlag, Berlin, 2009).

[48] K. Simons and D. Toomre, Nat. Rev. Mol. Cell Biol. 1, 31 (2000).

[49] M. Fallahi-Sichani and J. J. Linderman, PLoS One 4, e6604 (2009).

[50] L. J. Pike, J. Lipid Res. 44, 655 (2003).

[51] P. S. Niemelä, S. Ollila, M. T. Hyvönen, M. Karttunen, and I. Vattulainen, PLoS Comput. Biol. 3, e34 (2007).

[52] Y. H. Wu, X. S. Jin, O. Harrison, L. Shapiro, B. H. Honig, and A. Ben-Shaul, Proc. Natl. Acad. Sci. USA 107, 17592 (2010).

[53] L. LeBon, T. V. Lee, D. Sprinzak, H. Jafar-Nejad, and M. B. Elowitz, eLife 3, e02950 (2014).

[54] N. Gov, A. G. Zilman, and S. Safran, Phys. Rev. Lett. 90, 228101 (2003).

[55] S. A. Safran, N. Gov, A. Nicolas, U. S. Schwarz, and T. Tlusty, Phys. A (Amsterdam, Neth.) 352, 171 (2005).

[56] N. S. Gov and S. A. Safran, Biophys. J. 88, 1859 (2005).

[57] R. Rodríguez-García, I. López-Montero, M. Mell, G. Egea, N. S. Gov, and F. Monroy, Biophys. J. 108, 2794 (2015). 